\title{
Cognitive Load of Exercise Influences Cognition and Neuroplasticity of Healthy Elderly: An Exploratory Investigation
}

\author{
Yi-Ping Chao ${ }^{1,2}$ (D) Changwei W. Wu ${ }^{3,4} \cdot$ Li-Jung Lin $^{5} \cdot$ Cheng-Hung Lai ${ }^{6} \cdot$ Hong-Yi Wu ${ }^{7} \cdot$ Ai-Ling Hsu ${ }^{7}$. \\ Chiao-Nan Chen ${ }^{8}$
}

Received: 29 October 2019 / Accepted: 10 April 2020 / Published online: 30 April 2020

(c) The Author(s) 2020

\begin{abstract}
This study explored the effects of exercise with either high cognitive load or low cognitive load on cognitive performance and neuroplasticity in healthy elderly. Twenty-eight sedentary community-dwelling seniors participated in this study. Participants were assigned to one of three groups: the control group (C), low-cognitive load exercise group (LE), or highcognitive load exercise group (HE). Individuals in both exercise groups engaged in moderate-intensity aerobic exercise for 4 months. Resting-state functional MRI and diffusion MRI were used to investigate the effects of intervention on functional and structural connectivity, respectively. Analysis of covariance with baseline, age and the education year as covariates was used to determine the effects of intervention. We found 4 months of exercise with high-cognitive load, but not exercise with low-cognitive load, improved the overall cognitive function of healthy elderly. Additionally, the HE group showed increased resting-state functional connectivity of superior frontal gyrus and anterior cingulate cortex and decreased functional connectivity of middle occipital gyrus and postcentral gyrus. The age-related alterations in local efficiency and betweenness were protected by exercise. Our findings might suggest that exercise with greater cognitive load likely results in greater training effects on cognition and brain connectivity than exercise requiring lower cognitive loads for healthy elderly.
\end{abstract}

Keywords Dance $\cdot$ Diffusion tensor imaging $\cdot$ Functional connectivity $\cdot$ Plasticity

\section{Introduction}

Aging, which results in progressive decline in several functions after adulthood, has a pervasive and complex influence on physiology, cognition, and physical activity, and it has become a global issue in modern society. Age-related

Electronic supplementary material The online version of this article (https://doi.org/10.1007/s40846-020-00522-x) contains supplementary material, which is available to authorized users.

Chiao-Nan Chen

chiaonanchen@ym.edu.tw

1 Graduate Institute of Biomedical Engineering, Chang Gung University, Taoyuan, Taiwan

2 Department of Neurology, Chang Gung Memorial Hospital At Linkou, Taoyuan, Taiwan

3 Research Center of Brain and Consciousness, Shuang Ho Hospital, Taipei Medical University, Taipei, Taiwan

4 Graduate Institute of Humanities in Medicine, Taipei Medical University, Taipei, Taiwan decline in these functions not only reduces the quality of life and independence of elderly people, but also impacts the family, society, and national economy. Among the agerelated reductions of functionality, the decline in cognitive function among elderly people has received considerable attention. Cognitive function refers to brain functionality related to the formation of knowledge, attention, memory, judgment, language ability, computing power, space-time orientation ability, and reasoning ability [1]. The age-related cognitive decline is noticeable for the elderly and sometimes

5 Graduate Institute of Sport, Leisure and Hospitality Management, National Taiwan Normal University, Taipei, Taiwan

6 Graduate Institute of Rehabilitation Science, Chang Gung University, Taoyuan, Taiwan

7 Graduate Institute of Biomedical Electronics and Bioinformatics, National Taiwan University, Taipei, Taiwan

8 Department of Physical Therapy and Assistive Technology, National Yang- Ming University, 155, Sec. 2, Li-nong St, Taipei 112, Taiwan 
causes frustration, but it is different from dementia where the brain is damaged by diseases (such as cerebrovascular diseases or Alzheimer's disease) and with symptoms severe enough to interfere normal daily life.

Cognitive training involving the repetitive practice of cognitive tasks in a static condition is a approach to improve the cognition of elderly people [2]. Because of the association between age-related cognitive decline and cardiovascular diseases (hypertension, diabetes, and obesity), aerobic exercise that improves cardiovascular fitness and metabolic function has been proposed as an intervention to alleviate or even reverse age-related cognitive decline [3]. Nevertheless, interventional studies have provided inconsistent findings regarding the effects of aerobic exercise on the cognitive function of elderly people. Some studies have found that exercise improves the cognitive function of elderly people $[4,5]$, whereas others have found no differences in cognitive function between control and exercise intervention groups [6]. Meta-analyses have demonstrated that although aerobic exercise increases the cognitive function of healthy senior adults, the increase is not significant when compared with that in the control group [7, 8]. Training that combines physical and cognitive components provides greater benefit for cognition [9]. Exercise type, intensity, frequency, and duration all potentially influence training effects.

Neuroimaging studies have proposed that cognitive training and exercise improve the cognitive functions through neuroplasticity not only in patients with mild cognitive impairment but also in healthy sensor adults [10,11]. Moderate-intensity aerobic exercise modulates cognitively and clinically relevant functional brain networks in older adults, especially for increasing the integration of attention and executive control network (ECN) and the functional connectivity of default mode network (DMN) [12], and decreasing the brain atrophy in the anterior cingulate cortex (ACC) and medial temporal lobe [13]. Not only functional connectivity but also structural connectivity derived from diffusion tensor imaging (DTI) showed that physical exercise significantly increases the integrity of motor functioning related white matter fiber tracts [14]. Nevertheless, it is still unclear the effects of exercise with different cognitive load on neuroplasticity and cognitive function. The aim of this study was to explore the effects of exercise with either high cognitive load or low cognitive load on cognitive performance and neuroplasticity in healthy elderly. According to the published findings, we targeted the exercise-related neuroplasticity on the four specific networks: DMN, ECN, sensorimotor (SMN) and salience network (SN) encompassing ACC.

\section{Methods}

\subsection{Participants}

Twenty-eight sedentary community-dwelling senior adults (age $\geq 65$ years old) without known cognitive impairment were recruited. Volunteers were included if they were sedentary (exercised less than 90 min per week in the past year) and could walk at least $5 \mathrm{~m}$. Volunteers were excluded if they had diagnosed neurological and psychiatric disorders, had score of Mini Mental State Examination lower than 26, had heart failure or met the exclusion criteria specific to magnetic resonance imaging (MRI) scanning including claustrophobia and having metallic foreign body in the body. Participants were randomly assigned to either the high-cognitive load exercise group $(\mathrm{HE}, \mathrm{n}=10)$ or the low-cognitive load exercise group (LE, $n=9)$. For the allocation, lots were drawn by a person who did not involve in this study. Participants who registered after the start of the exercise program were allocated to the control group $(\mathrm{C}, \mathrm{n}=9)$. All participants were assessed at baseline and after 4 months of intervention. This study was approved by the Institutional Review Board of Chang Gung Medical Foundation and all methods were performed in accordance with the relevant guidelines and regulations. Written informed consents were obtained from all the participants of this study. Information that could lead to identification of a participant (e.g. clinical images and videos) was not collected in this study.

\subsection{Exercise Pogram}

Participants in the HE and LE groups engaged in moderate-intensity aerobic exercise training for 4 months; exercise frequency was three times a week, and each session lasted for $50 \mathrm{~min}$. The exercise mode of the LE group was walking on a treadmill supervised by a physical therapist. Walking was chosen because it is a widely accepted exercise type among elderly people. The exercise mode of the HE group was aerobic dance led by an aerobic dance instructor. The dance program included common aerobic movements, such as V step, marching, kicks, and lunges in different directions. Participants followed the movement of the instructor, learnt new dance movements, memorized the order of different movements, and switched between movements throughout the exercise. In both groups, each 50-min exercise session comprised $10 \mathrm{~min}$ of warm-up, 30 min of moderate-intensity exercise (55\% to $69 \%$ of the age-predicted maximal heart rate), and $10 \mathrm{~min}$ of cooldown. To monitor equal exercise intensity between LE and HE groups, all participants wore a heart rate monitor 
(Polar RS400; Polar Electro Inc.) during exercise. The average heart rate during main exercise was similar between the LE and HE groups (Supplementary Table).

\subsection{Measurement}

\subsubsection{Cognitive Performance}

Cognitive performance of participants was assessed using the Chinese version of Cognitive Abilities Screening Instrument 2.0 (CASI 2.0). CASI is a 100-point scale that assesses nine domains of cognitive function, including attention, orientation, short-term memory, long-term memory, language, drawing, abstract thinking and judgment, mental manipulation, and animal name fluency. Higher scores indicate higher cognitive function $[1,15]$. The total score and the domain scores of CASI was used to evaluate the cognitive ability of individuals. The total score reflects the overall cognitive ability of individuals and the nine domain scores reflect the ability of designated individual cognitive abilities. The validity of the overall score and domain scores of CASI has been confirmed previously [16, 17]. We also recorded the time taken to finish the cognitive test because time it takes to respond to questions reflects the attention, processing speed and executive function of individuals [18].

\subsubsection{MRI Acquisition}

MRI data were acquired on a $3 \mathrm{~T}$ scanner (Prisma, Siemens, Erlangen, Germany) with a 20-channel head coil at National Taiwan University. High-resolution longitudinal relaxation time weighted imaging (T1-weighted anatomical images) were collected using a magnetization-prepared rapid gradient-echo (MP-RAGE) sequence: inversion time $(\mathrm{TI})=900 \mathrm{~ms}$, repetition time $(\mathrm{TR})=2000 \mathrm{~ms}$, echo time $(\mathrm{TE})=2.28 \mathrm{~ms}$, flip angle $(\mathrm{FA})=8^{\circ}$, field-of-view $($ FOV $)=256 \times 256 \mathrm{~mm}^{2}$, voxel size $=1 \times 1 \times 1 \mathrm{~mm}^{3}$, slice number $=192$, and acquisition time $=277 \mathrm{~s}$. Resting-state functional MRI (RS-fMRI) data were acquired using a gradient-echo planar imaging (EPI) sequence with whole-brain coverage: $\mathrm{FOV}=192 \times 192 \mathrm{~mm}^{2}$, slice number $=32$, voxel size $=3 \times 3 \times 4 \mathrm{~mm}^{3}, \mathrm{TR}=2000 \mathrm{~ms}, \mathrm{TE}=32 \mathrm{~ms}, \mathrm{FA}=84^{\circ}$ and total scan time $=286 \mathrm{~s}$. In RS-fMRI, participants were instructed to immobilize head position, to visually fixate on a cross, not to fall asleep, and not to think of anything in particular. Diffusion tensor images (DTI) were acquired using a dual-spin-echo EPI sequence: slice number $=55$ with thickness $=2.5 \mathrm{~mm}$ without gap, FOV $=240 \times 240 \mathrm{~mm}^{2}$, voxel size $=2.5 \mathrm{~mm}$ isotropic, $\mathrm{TR} / \mathrm{TE}=7000 / 87 \mathrm{~ms}$, and b-value $=1000 \mathrm{~s} / \mathrm{mm}^{2}$ with 30 noncollinear diffusion gradient directions and 10 null images $(b=0)$. Total scan time for DTI acquisition was $280 \mathrm{~s}$.

\subsubsection{MRI Preprocessing}

In network analyses, we parcellated both fMRI and DTI data into 90 brain regions using Automated Anatomical Labeling (AAL) [19]. First, for functional network construction, the RS-fMRI data were preprocessed using the default preprocessing pipeline of CONN functional connectivity toolbox (ver.15 h) [20]. After preprocessing, the average fMRI signal of the white matter and cerebrospinal fluid (CSF) was used for nuissance regression and underwent the band pass filter $(0.01-0.08 \mathrm{~Hz})$. Finally, the correlation between the time courses each pair of brain parcellations was computed for each volunteer, generating the weighted connectivity matrix. Second, for structural network construction, the DTI data were processed using pipeline for analyzing brain diffusion images (PANDA) (ver.1.3.1) [21]. Based on matrix laboratory (MATLAB) and FSL (Functional Magnetic Resonance Imaging of the Brain Software Library). For fiber tracking, fiber assignment was performed using a fiber assignment by continuous tracking (FACT) algorithm, with an FA threshold of 0.2 and an angle threshold of 45 . For network construction, the 90 AAL regions were taken as network nodes. Finally, two weighted and undirected connectivity matrices of fiber number (FN) and FA values were generated for each participant.

\subsubsection{Functional and Structural Connectivity}

The network measures were computed using Brain Connectivity Toolbox (BCT) [22]. In BCT, we estimated the network measures using the weighted matrices over a range of thresholds (FN in structural connectivity: 0-10, 1 increments; matrix density in functional connectivity: $0.37-0.5$, 0.001 increments) [23-25]. After thresholding, the weighted FN matrix became a binary matrix. New weighted matrix was then generated by multiplying the FA-based connectivity matrix by the binary matrix. The generated binary and weighted matrices (for both structural and functional connectivity) were calculated for further network analyses. For these analyses, three types of local node measures were selected: (1) degree: the number of node connections; (2) betweenness: the fraction of all shortest paths in the network that contains a given node; (3) local efficiency: the global efficiency computed from the reciprocal of path lengths to the neighbors of a given node [22].

\subsection{Statistical Analysis}

All data are presented as mean \pm SEM or number (percentage). SPSS version 24 (SPSS, Chicago, Illinois, USA) and GraphPad Prism 5.0 (GraphPad software Inc., La Jolla, CA, USA) were used to analyze data and draw the plots. Baseline differences among the three groups were tested using 
one-way analysis of variance (ANOVA) and chi-squared tests. Analysis of covariance (ANCOVA) with post-test values as the dependent variable, and baseline, age and the education year (note: age and education are two predictors of the CASI score [15]) as covariates was used to determine the effects of intervention and the Fisher's Least Significant Difference was used as the post-hoc test. Pearson's correlation coefficient was used to examine the association between outcome variables. $\mathrm{P}<0.05$ was considered significant for all variables except outcomes of brain plasticity where the significance level was set at 0.013 for Bonferroni correction (two-tailed) for multiple comparisons regarding four targeting brain networks were explored.

\section{Results}

\subsection{Baseline Characteristics}

The study population was characterized by a mean age of $69.2 \pm 0.8$ years (range 63 to 79 years), years of education of $10.5 \pm 0.8$, BMI of $25.0 \pm 0.5 \mathrm{~kg} / \mathrm{m}^{2}$, and waist circumference of $88.6 \pm 1.6 \mathrm{~cm}$ for men and of $85.3 \pm 1.3 \mathrm{~cm}$ for women. Twenty-one percent of participants had hypertension, and $7 \%$ had diabetes. Regarding cognitive performance, the study population had a total CASI score of $91.0 \pm 0.8$, long-term memory domain score of $9.96 \pm 0.04$, short-term memory domain score of $10.57 \pm 0.21$, attention domain score of $7.64 \pm 0.21$, mental manipulation domain score of $7.93 \pm 0.30$, orientation domain score of $17.61 \pm 0.15$, abstract thinking and judgment domain score of $10.11 \pm 0.28$, language domain score of $9.25 \pm 0.18$, drawing domain score of $10.00 \pm 0.00$, and animal name fluency domain score of $7.93 \pm 0.34$. The baseline characteristics of participants in the $\mathrm{C}, \mathrm{LE}$, and HE groups were all similar, except for years of education. Participants in the LE and HE groups had more years of education than those in the $\mathrm{C}$ group, but no significant difference was observed between the LE and HE groups (Table 1).

\subsection{Exercise Compliance}

Two participants in the HE group and one participant in the LE group withdrew from the study before the post-test. Specifically, one participant in the HE group withdrew in the first month of training due to kidney stone surgery, and the other participant withdrew due to frequent colds. Contact with one participant in the LE group was lost after baseline assessment. The average exercise attendance rate of participants who completed both pre- and post-tests was $90.0 \%$ for the HE group and $84.1 \%$ for the LE group. No significant difference was observed in the attendance rate of exercise training between the HE and LE groups $(P=0.21)$.
Table 1 Baseline characteristics of participants

\begin{tabular}{llll}
\hline & $\mathrm{C}(\mathrm{n}=9)$ & $\mathrm{LE}(\mathrm{n}=9)$ & $\mathrm{HE}(\mathrm{n}=10)$ \\
\hline Male: Female & $4: 5$ & $4: 5$ & $4: 6$ \\
Age (years) & $67.7 \pm 1.1$ & $71.2 \pm 1.4$ & $68.8 \pm 1.3$ \\
Education (years) & $6.7 \pm 1.1$ & $13.8 \pm 0.7^{*}$ & $11 \pm 1.1^{*}$ \\
Hypertension, $\mathrm{n}(\%)$ & $1(11)$ & $3(33)$ & $2(20)$ \\
Diabetes, $\mathrm{n}(\%)$ & $0(0)$ & $0(0)$ & $2(20)$ \\
Body mass index $\left(\mathrm{kg} / \mathrm{m}^{2}\right)$ & $25.7 \pm 0.6$ & $25.1 \pm 0.7$ & $23.8 \pm 1.0$ \\
CASI $(100)$ & $88.8 \pm 2.1$ & $92.1 \pm 0.9$ & $92.5 \pm 0.9$ \\
\hline
\end{tabular}

Data are mean \pm SEM; $* P<0.05$ compared with the control group; $C$ control group; $C A S I$ total score of cognitive abilities screening instrument; $H E$ high-cognitive load exercise group; $L E$ low-cognitive load exercise group

\subsection{Effects of Exercise Intervention}

\subsubsection{Cognitive Performance}

The total CASI score of HE group at post-test was significantly higher than that of the $\mathrm{C}$ group after adjusting the baseline values, age and the education $(P<0.05)$. There were no significant differences in the total CASI score at post-test between $\mathrm{C}$ and LE groups and between LE and HE groups. The HE group took shorter time to finish the cognitive battery at post-test than the C and LE groups (Table 2).

\subsubsection{Functional and Structural Connectivity}

Network analysis of functional connectivity (based on RSfMRI data) showed group-dependent changes from pretest to post-test (Fig. 1a). Specifically, HE group had increased degrees in the left superior frontal gyrus (SFG, AAL region 3) from $E C N$ and left anterior cingulate cortex (ACC, AAL region 31) from $\mathrm{SN}$, and had decreased degrees in the left middle occipital gyrus (MOG, AAL region 51) from the visual network, and left postcentral gyrus (PCG, AAL region 57) from SMN after the 4-month intervention (Fig. 1b-e). Moreover, the local efficiency of the left angular gyrus (AG, AAL region 65) from DMN was significantly increased in the $\mathrm{C}$ group, but not in the LE and HE groups. The local efficiency of left inferior temporal gyrus (ITG, AAL region 89), sub-region of DMN, significantly decreased in the HE group, but not in the $\mathrm{C}$ and LE groups (Fig. 1f-g). Network analysis of structural connectivity demonstrated an alternative scenario (Fig. 2a). Specifically, the betweenness measure of the left middle temporal gyrus (MTG, AAL region 85) from DMN derived from binary connectivity matrices alone and the left ITG (AAL region 89) derived from weighted matrices showed significant increase in the $\mathrm{C}$ group after the 4 months (Fig. 2b-c). 
Table 2 CASI scores of each group before and after exercise intervention

\begin{tabular}{|c|c|c|c|c|c|c|c|c|}
\hline & \multicolumn{2}{|l|}{$\mathrm{C}$} & \multicolumn{2}{|l|}{ LE } & \multicolumn{2}{|l|}{$\mathrm{HE}$} & \multirow{2}{*}{$\begin{array}{l}P \text { value of } \\
\text { ANCOVA }\end{array}$} & \multirow{2}{*}{$\begin{array}{l}\text { Difference } \\
\text { between } \\
\text { groups }\end{array}$} \\
\hline & Pre-test & Post-test & Pre-test & Post-test & Pre-test & Post-test & & \\
\hline CASI (100) & $88.8 \pm 2.1$ & $89.2 \pm 1.9$ & $92.1 \pm 0.8$ & $94.9 \pm 0.8^{*}$ & $92.5 \pm 1.1$ & $95.5 \pm 1.6^{*}$ & 0.048 & $\mathrm{C}<\mathrm{HE}$ \\
\hline $\begin{array}{l}\text { Time to finish the } \\
\text { test }(\mathrm{sec})\end{array}$ & $570 \pm 24$ & $560 \pm 21$ & $553 \pm 16$ & $529 \pm 9$ & $574 \pm 18$ & $517 \pm 12 *$ & 0.004 & $\mathrm{C}, \mathrm{LE}>\mathrm{HE}$ \\
\hline LTM (10) & $9.9 \pm 0.1$ & $10 \pm 0.0$ & $10 \pm 0.0$ & $10 \pm 0.0$ & $10 \pm 0.0$ & $10 \pm 0.0$ & & \\
\hline STM (12) & $10.1 \pm 0.5$ & $10 \pm 0.3$ & $10.8 \pm 0.5$ & $11.1 \pm 0.3$ & $10.9 \pm 0.2$ & $11.3 \pm 0.4$ & 0.367 & \\
\hline ATTEN (8) & $7.7 \pm 0.4$ & $7.6 \pm 0.4$ & $7.5 \pm 0.3$ & $7.4 \pm 0.3$ & $7.4 \pm 0.4$ & $7.5 \pm 0.4$ & 0.363 & \\
\hline MENMA (10) & $7.7 \pm 0.5$ & $7.7 \pm 0.5$ & $8 \pm 0.6$ & $8.9 \pm 0.5$ & $8.5 \pm 0.6$ & $8.8 \pm 0.6$ & 0.924 & \\
\hline ORIEN (18) & $17.3 \pm 0.4$ & $17.3 \pm 0.4$ & $17.9 \pm 0.1$ & $18 \pm 0.0$ & $17.8 \pm 0.2$ & $17.9 \pm 0.1$ & 0.895 & \\
\hline ABSTR (12) & $9.6 \pm 0.7$ & $9.7 \pm 0.6$ & $10.3 \pm 0.2$ & $10.4 \pm 0.3$ & $10.8 \pm 0.5$ & $10.9 \pm 0.3$ & 0.526 & \\
\hline LANG (10) & $9.2 \pm 0.5$ & $9.2 \pm 0.5$ & $9.3 \pm 0.3$ & $9.6 \pm 0.3$ & $9.3 \pm 0.2$ & $9.9 \pm 0.1^{*}$ & 0.071 & \\
\hline DRAW (10) & $10 \pm 0.0$ & $10 \pm 0.0$ & $10 \pm 0.0$ & $10 \pm 0.0$ & $10 \pm 0.0$ & $10 \pm 0.0$ & & \\
\hline ANML (10) & $7.3 \pm 0.7$ & $7.8 \pm 0.5$ & $8.5 \pm 0.7$ & $9.5 \pm 0.3$ & $8 \pm 0.6$ & $9.4 \pm 0.5$ & 0.081 & \\
\hline
\end{tabular}

Data are mean \pm SEM; $* P<0.001$ compared to the pre-test scores of the same group; $A B S T R$ abstract thinking and judgment; ANML animalname fluency; $A T T E N$ attention; $C$ control group; $C A S I$ total score of cognitive abilities screening instrument; $D R A W$ drawing; $H E$ high-cognitive load exercise group; $L A N G$ language; $L E$ low-cognitive load exercise group; LTM long term memory; MENMA mental manipulation; ORIEN:orientation; STM short term memory
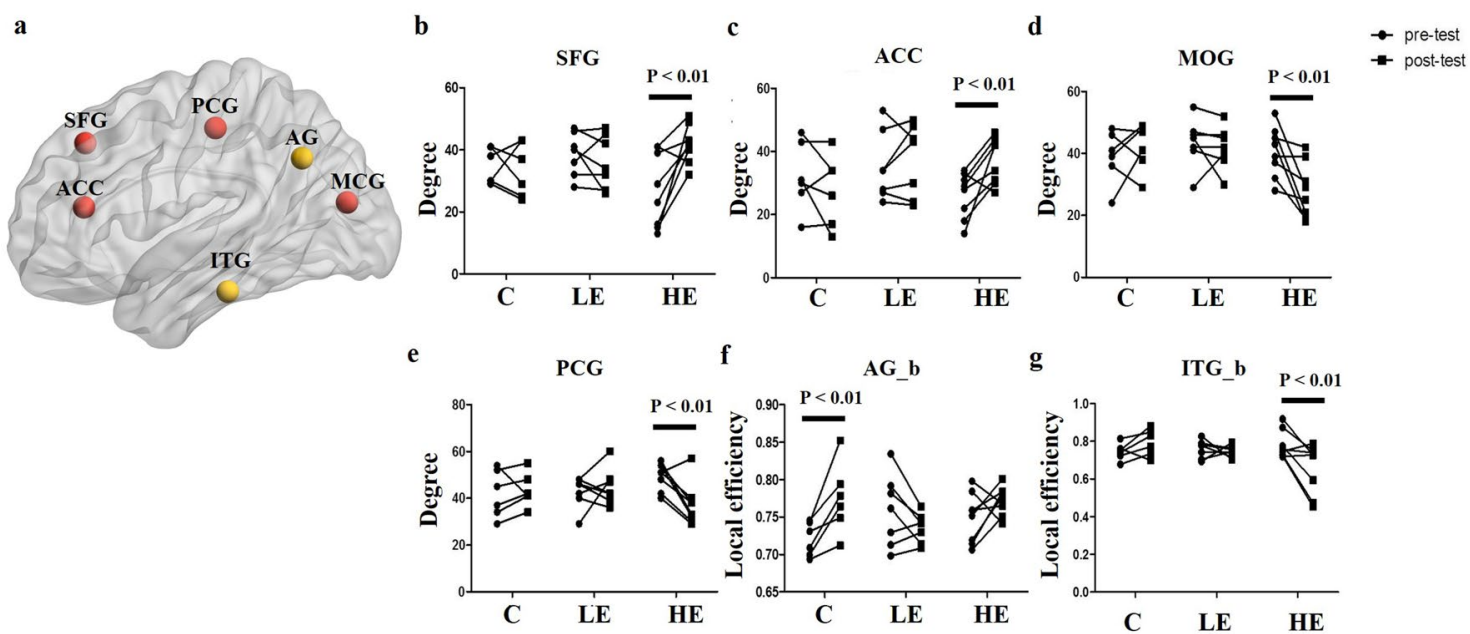

Fig. 1 Brain regions showing group-dependent neuroplasticity in functional connectivity. a The summary figure of brain regions showing group-dependent neuroplasticity in functional connectivity (red nodes indicate degree, and yellow nodes indicate local efficiency). b-g Network measures of brain regions showing group-dependent neuroplasticity in functional connectivity. Each line links the pre-

\subsubsection{Relationship Between Changes in Cognitive Performance and Brain Plasticity}

The changes in the time to complete the cognitive performance test were negatively correlated with the changes in ACC degree in functional connectivity and were positively correlated with the changes in MOG degree in functional connectivity and the changes in MTG betweenness

and post-test data of each individual. $A C C$ anterior cingulate cortex; $A G$ angular gyrus; $C$ control group; $H E$ high-cognitive load exercise group; ITG inferior temporal gyrus; $L E$ low-cognitive load exercise group; $M O G$ middle occipital gyrus; $S F G$ superior frontal gyrus; $P C G$ postcentral gyrus; $b$ binary matrix $(\mathrm{n}=6$ in the $\mathrm{C}$ group; $\mathrm{n}=7$ in the LE group; $n=7$ in the HE group)

in structural connectivity. The changes in the total CASI score were negatively correlated with the changes in AG local efficiency in functional connectivity and the changes in MTG betweenness in structural connectivity. Changes in the abstract thinking and judgment domain score were negatively correlated with the changes in PCG local efficiency in functional connectivity. Changes in the animal name fluency domain score were negatively correlated 

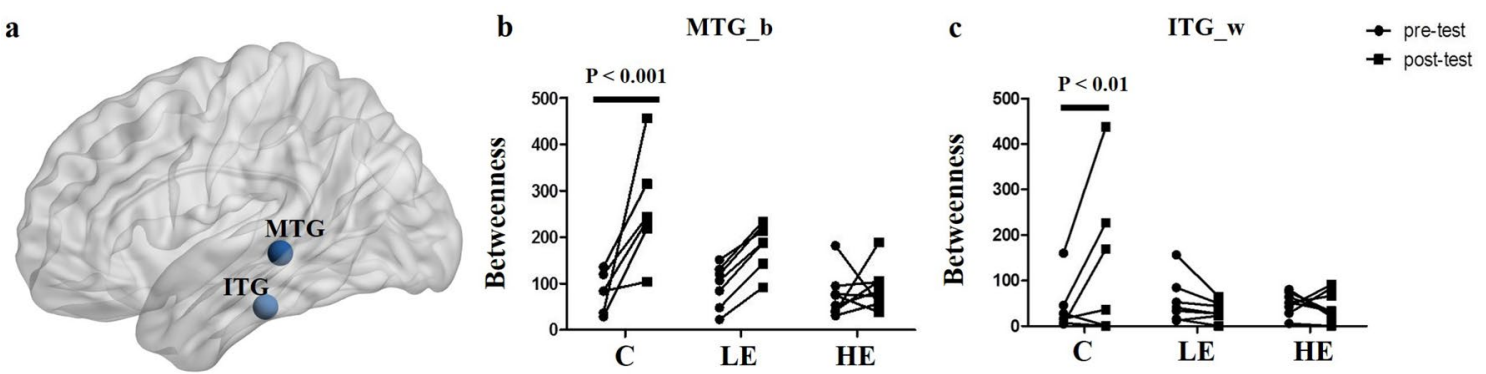

Fig. 2 Brain regions showing group-dependent neuroplasticity in structural connectivity. a The summary figure of brain regions showing group-dependent neuroplasticity in structural connectivity (blue nodes indicate betweenness). b-c Network measures of brain regions showing group-dependent neuroplasticity in structural connectiv-

with the changes in ITG local efficiency in functional connectivity (Table 3 ).

\section{Discussion}

The main finding of this study was that 4 months of exercise with high-cognitive load, but not exercise with low-cognitive load, improved the overall cognitive function of healthy elderly. MRI indices provided neuroscientific evidence for differential training effects between exercise with high and low cognitive loads. Exercise-related changes in functional connectivity were only prominent in the HE group. To the network specificity, the degrees of ECN and SN changed in the HE group after intervention. In addition, exercise prevented time-related changes in structural betweenness in the C group.

The novel finding of this study is that at the same exercise intensity, duration, and frequency, exercise involving high cognitive loads (dancing) had a more positive influence on ity. Each line links the pre- and post-test data of each individual. $C$ control group; $H E$ high cognitive load exercise group; ITG inferior temporal gyrus; $L E$ low cognitive load exercise group; $M T G$ middle temporal gyrus; $b$ binary matrix; $w$ weighted matrix $(\mathrm{n}=6$ in the $\mathrm{C}$ group; $n=7$ in the LE group; $n=7$ in the HE group)

cognitive function than exercise involving low cognitive loads (treadmill walking). Dancing is a cognitively taxing activity because this sensorimotor activity involves acoustic stimulation and various cognitive function such as attention, language, memory, visuo-spatial ability and visuomotor integration [26, 27]. Greater improvement in the executive function of healthy elderly people has been shown after one session of aerobic dance requiring higher cognitive engagement than after one session of aerobic dance requiring lower cognitive engagement [28]. Anderson-Hanley et al. found greater improvements in the executive function of elderly people with cyber cycling, where participants received more cognitive stimuli, than elderly people who rode traditional stationary bikes [29]. Our findings together with those of previous studies suggest that to improve the cognitive function through exercise, older individuals should choose an exercise type with at least moderate intensity and with high cognitive loads.

By quantitative MRI technologies, we found the beneficial effects of high-cognitive load exercise associated with

Table 3 The relationship between changes of cognitive performances and brain plasticity

\begin{tabular}{llrrrrrrrrrrrr}
\hline & & Time & CASI & LTM & STM & ATTEN & MENMA & ORIEN & ABSTR & LANG & DRAW & ANML \\
\hline Degree & SFG (FC) & -.423 & 0.069 & -.167 & .229 & .400 & -.425 & -.043 & -.012 & .270 &.$^{\mathrm{a}}$ & .132 \\
& ACC (FC) & $-.446^{*}$ & .079 & -.070 & -.119 & .323 & -.241 & .092 & -.021 & .346 &.$^{\mathrm{a}}$ & .232 \\
& MOG (FC) & $.502^{*}$ & -.061 & -.052 & -.032 & -.331 & .376 & -.105 & -.121 & -.260 &.$^{\mathrm{a}}$ & -.116 \\
& PCG (FC) & .283 & -.148 & .171 & .029 & -.279 & .260 & .203 & $-.479^{*}$ & -.130 &.$^{\mathrm{a}}$ & -.151 \\
Local Efficiency & AG (FC_b) & -.089 & $-.461^{*}$ & .229 & -.162 & .031 & -.435 & -.146 & .064 & -.048 &.$^{\mathrm{a}}$ & -.207 \\
& ITG (FC_b) & .329 & -.124 & .186 & .114 & -.431 & .263 & -.097 & .136 & -.197 &.$^{\mathrm{a}}$ & $-.445^{*}$ \\
Betweenness & ITG (SC_w) & .236 & -.357 & -.069 & -.069 & -.054 & -.103 & -.089 & -.225 & -.270 &.$^{\mathrm{a}}$ & -.117 \\
& MTG (SC_b) & $.527^{*}$ & $-.470^{*}$ & .191 & -.296 & .015 & -.110 & -.320 & -.275 & -.269 &.${ }^{\mathrm{a}}$ & -.081 \\
\hline
\end{tabular}

${ }^{*} \mathrm{P}<0.05 ; a$ can not be computed because all participants received full scores in the drawing domain; $b$ binary matrix; $w$ weighted matrix; $A C C$ anterior cingulate cortex; $A B S T R$ abstract thinking and judgment; $A G$ angular gyrus; $A N M L$ animal-name fluency; $A T T E N$ attention; $C A S I$ total score of cognitive abilities screening instrument; $D R A W$ drawing; $F C$ functional connectivity based on RS-fMRI data; ITG inferior temporal gyrus; $L A N G$ language; $L T M$ long term memory; $M E N M A$ mental manipulation; $M O G$ middle occipital gyrus; $M T G$ middle temporal gyrus; ORIEN orientation; PCG postcentral gyrus; $S C$ structural connectivity based on DTI data; SFG superior frontal gyrus; STM short term memory 
subregions of ECN, DMN, SN and SMN in well agreement with previous findings. However, because these regions may involve cross-network functionality following the exercise intervention, the functional roles of each subregion are discussed below. We found that high-cognitive load exercise increased resting-state functional connectivity in SFG and $\mathrm{ACC}$ and decreased functional connectivity in sensory-associated brain regions, MOG and PCG. The SFG and ACC are associated with focusing attention and working memory and are highly susceptible to age-related alterations in cognitive engagement and functional connectivity [30, 31]. The role of SFG in working memory was evidenced by a lesion study, in which patients with brain lesions restricted to the left SFG had significant deficits in working memory compared with healthy controls and patients with prefrontal lesions but intact SFG, particularly when the complexity of the tasks increased [32]. The ACC is responsible for the selection of salient stimuli and the reactivity of individuals to affective inputs [33]. Greater ACC activity predicts higher focusing attention, particularly during distracting events [34]. MOG and PCG are perception-related brain regions for vision and limb sensation, respectively. The decreased functional connectivity of MOG and PCG in the HE group implied that training involving high cognitive engagement decreased the interference originating from visual and physical perceptions. Taken together, the findings indicated that the increased functional connectivity of SFG and ACC and the decreased functional connectivity of MOG and PCG in the HE group potentially promoted the ability to focus in elderly participants.

Our findings for the exercise-induced brain plasticity of SFG and ACC are similar to those of previous studies. Colcombe et al. showed that 6 months of moderate-to-high intensity exercise training increased the brain volume of the ACC, supplementary motor area, and middle frontal gyrus [35]. Voss et al. showed that a 1-year walking intervention program increased the functional connectivity of the frontal executive network in elderly people. However, the exercise-induced brain plasticity was not significant within 6 months of intervention [36]. In the current study, 4 months of moderate-intensity exercise involving high cognitive loads resulted in favorable changes in functional connectivity among elderly participants. Therefore, the characteristics of exercise affect the length of training required to observe significant brain plasticity. Exercise involving high cognitive load may result in rapid neuroplasticity effects, leading to subsequent cognitive improvements in senior adults.

We found that exercise, particularly high-cognitive load exercise protected the brain from the age-related increase in local efficiency in AG and ITG. In addition, the changes in AG local efficiency were negatively correlated with the changes in the total CASI score. Both cross-sectional and longitudinal studies have shown that aging leads to increases in brain local efficiency $[37,38]$. The beneficial effects of exercise on local efficiency have been reported previously; that is, the functional connectivity of AG did not change in the control group but decrease in elderly people who engaged 6 months of multimodal activities, including cognitive, aerobic, and recreational activities [39].

Regarding structural connectivity, the betweenness of MTG and ITG increased in the control group, but not in the high-cognitive load exercise group. Changes in the betweenness of MTG were negatively associated with the changes in cognitive performance. The betweenness of a brain area is the fraction of the number of shortest paths connecting neighboring brain areas that pass through the specific brain area. Greater betweenness of a brain area suggests that certain brain areas participate in a greater number of shortest paths. The betweenness of a brain region increases when the brain region has more linkage with other brain regions or when its neighboring brain region is lost [40]. Regarding the inverse correlation between the exercise-related changes in betweenness and cognition of our elderly population, our finding of the increased betweenness of MTG and ITG in the control group was likely due to the loss of gray matter volume. This finding was supported by additional analytic results that showed gray matter volumes of MTG and ITG were decreased in the control group, but no significant changes in the gray matter volume were found in the LE and HE groups (data not shown). The gray matter volume of the ITG has been reported to decrease with aging [41]. In brief, our findings suggest that high-cognitive load exercise improves the cognitive function of elderly people by preventing the volumetric loss of the temporal gyrus and its corresponding maintenance in betweenness.

This study had some limitations. First, this study had small sample size with $9 \sim 10$ subjects in each group. Further studies with larger sample sizes are needed. Second, this study targeted on specific networks and their subregions associated with exercise, which involves multiple comparison issue in statistics. We took the network number into account for the multiple comparison for disclosing the plausible network changes. Given sufficient sample size, future studies are warranted to validate our findings. Third, while subjects in two exercise groups were allocated randomly, the control group was not allocated in a randomized manner. Although the recruitment strategies of the control group were not different from the other two groups, the education year was fewer in the control group than the other two groups. The difference in the education year among groups was likely due to the small sample size of the exploratory study. Thus, our finding could be influenced by the difference in the education between the control group and exercise groups even though education has been adjusted in ANCOVA as a covariate in this study. Last, this study did 
not have a group with only cognitive training, thus limiting the interpretation of the results.

In conclusion, this study might suggest that exercise with greater cognitive load likely results in greater training effects on cognition and brain connectivity than exercise requiring lower cognitive loads for healthy elderly.

Acknowledgements We thank all the subjects who volunteered to participate this study. This work was supported by Ministry of Science and Technology (MOST 104-2410-H-182-026, MOST 105-2628-B-038-013-MY3 \& MOST 108-2321-B-038-005-MY2), Chang Gung Memorial Hospital (BMRPB41, BMRPC78) and National Health Research Institutes (NHRI-EX108-10829-EI). We also thank the MOST-supported Imaging Center for Integrated Body, Mind and Culture Research, National Taiwan University for technical and facility supports. This manuscript was edited by Wallace Academic Editing.

Author Contributions Chen CN, Chao YP and Wu CW provided idea/ research design, project management and facilities/equipment. Chen $\mathrm{CN}$, Lai $\mathrm{CH}$, Lin $\mathrm{LJ}, \mathrm{Wu} \mathrm{HY}$ and Hsu AL provided data collection, analysis and interpretation of data. Chen $\mathrm{CN}$, Chao $\mathrm{YP}$ and $\mathrm{Wu} \mathrm{CW}$ interpreted the data and wrote the manuscript. All authors reviewed and approved the manuscript.

\section{Compliance with Ethical Standards}

Conflict of interests The authors declare that they have no competing interests.

Open Access This article is licensed under a Creative Commons Attribution 4.0 International License, which permits use, sharing, adaptation, distribution and reproduction in any medium or format, as long as you give appropriate credit to the original author(s) and the source, provide a link to the Creative Commons licence, and indicate if changes were made. The images or other third party material in this article are included in the article's Creative Commons licence, unless indicated otherwise in a credit line to the material. If material is not included in the article's Creative Commons licence and your intended use is not permitted by statutory regulation or exceeds the permitted use, you will need to obtain permission directly from the copyright holder. To view a copy of this licence, visit http://creativecommons.org/licenses/by/4.0/.

\section{References}

1. Lin, K. N., Wang, P. N., Liu, C. Y., Chen, W. T., Lee, Y. C., \& Liu, H. C. (2002). Cutoff scores of the cognitive abilities screening instrument, Chinese version in screening of dementia. Dementia and Geriatric Cognitive Disorders, 14(4), 176-182.

2. Kelly, M. E., Loughrey, D., Lawlor, B. A., Robertson, I. H., Walsh, C., \& Brennan, S. (2014). The impact of cognitive training and mental stimulation on cognitive and everyday functioning of healthy older adults: a systematic review and meta-analysis. Ageing Research Reviews, 15, 28-43.

3. Meyer, J. S., Rauch, G., Rauch, R. A., \& Haque, A. (2000). Risk factors for cerebral hypoperfusion, mild cognitive impairment, and dementia. Neurobiology of Aging, 21(2), 161-169.

4. Fabre, C., Chamari, K., Mucci, P., Masse-Biron, J., \& Prefaut, C. (2002). Improvement of cognitive function by mental and/or individualized aerobic training in healthy elderly subjects. International Journal of Sports Medicine, 23(6), 415-421.
5. Smith, J. C., Nielson, K. A., Antuono, P., Lyons, J. A., Hanson, R. J., Butts, A. M., et al. (2013). Semantic memory functional MRI and cognitive function after exercise intervention in mild cognitive impairment. Journal of Alzheimer's Disease, 37(1), 197-215.

6. Legault, C., Jennings, J. M., Katula, J. A., Dagenbach, D., Gaussoin, S. A., Sink, K. M., et al. (2011). Designing clinical trials for assessing the effects of cognitive training and physical activity interventions on cognitive outcomes: the Seniors Health and Activity Research Program Pilot (SHARP-P) study, a randomized controlled trial. BMC Geriatrics, 11, 27.

7. Kelly, M. E., Loughrey, D., Lawlor, B. A., Robertson, I. H., Walsh, C., \& Brennan, S. (2014). The impact of exercise on the cognitive functioning of healthy older adults: A systematic review and meta-analysis. Ageing Research Reviews, 16c, 12-31.

8. Angevaren, M., Aufdemkampe, G., Verhaar, H. J., Aleman, A., \& Vanhees, L. (2008). Physical activity and enhanced fitness to improve cognitive function in older people without known cognitive impairment. Cochrane Database of Systematic Reviews (3), Cd005381.

9. Levin, O., Netz, Y., \& Ziv, G. (2017). The beneficial effects of different types of exercise interventions on motor and cognitive functions in older age: A systematic review. European Review of Aging and Physical Activity, 14(1), 20.

10. Antonio, J., Simon-Pearson, L., Goldberg, T., Sneed, J. R., Rushia, S., Kerner, N., et al. (2019). Cognitive training and neuroplasticity in mild cognitive impairment (COG-IT): protocol for a two-site, blinded, randomised, controlled treatment trial. British Medical Journal Open, 9(8), e028536.

11. Deng, L., Cheng, Y., Cao, X., Feng, W., Zhu, H., Jiang, L., et al. (2019). The effect of cognitive training on the brain's local connectivity organization in healthy older adults. Scientific Reports, 9(1), 9033.

12. Weng, T. B., Pierce, G. L., Darling, W. G., Falk, D., Magnotta, V. A., \& Voss, M. W. (2017). The acute effects of aerobic exercise on the functional connectivity of human brain networks. Brain Plasticity, 2(2), 171-190.

13. Li, M. Y., Huang, M. M., Li, S. Z., Tao, J., Zheng, G. H., \& Chen, L. D. (2017). The effects of aerobic exercise on the structure and function of DMN-related brain regions: a systematic review. International Journal of Neuroscience, 127(7), 634-649.

14. Svatkova, A., Mandl, R. C. W., Scheewe, T. W., Cahn, W., Kahn, R. S., \& Hulshoff Pol, H. E. (2015). Physical exercise keeps the brain connected: biking increases white matter integrity in patients with schizophrenia and healthy controls. Schizophrenia Bulletin, 41(4), 869-878.

15. McCurry, S. M., Edland, S. D., Teri, L., Kukull, W. A., Bowen, J. D., McCormick, W. C., et al. (1999). The cognitive abilities screening instrument (CASI): data from a cohort of 2524 cognitively intact elderly. International Journal of Geriatric Psychiatry, 14(10), 882-888.

16. Tsai, R. C., Lin, K. N., Wang, H. J., \& Liu, H. C. (2007). Evaluating the uses of the total score and the domain scores in the Cognitive Abilities Screening Instrument, Chinese version (CASI C-2.0): results of confirmatory factor analysis. International Psychogeriatrics, 19(6), 1051-1063.

17. Lin, K. N., Wang, P. N., Liu, H. C., \& Teng, E. L. (2012). Cognitive Abilities Screening Instrument, Chinese Version 2.0 (CASI C-2.0): administration and clinical application. Acta Neurol Taiwan, 21(4), 180-189.

18. OIDAP (Occupational Information Development Advisory Panel) (2009). Mental cognitive subcommittee: Content model and classification recommendations.

19. Tzourio-Mazoyer, N., Landeau, B., Papathanassiou, D., Crivello, F., Etard, O., Delcroix, N., et al. (2002). Automated anatomical labeling of activations in SPM using a macroscopic anatomical 
parcellation of the MNI MRI single-subject brain. Neuroimage, 15(1), 273-289.

20. Whitfield-Gabrieli, S., \& Nieto-Castanon, A. (2012). Conn: a functional connectivity toolbox for correlated and anticorrelated brain networks. Brain Connectivity, 2(3), 125-141.

21. Cui, Z., Zhong, S., Xu, P., He, Y., \& Gong, G. (2013). PANDA: A pipeline toolbox for analyzing brain diffusion images. Frontiers in Human Neuroscience, 7, 42.

22. Rubinov, M., \& Sporns, O. (2010). Complex network measures of brain connectivity: uses and interpretations. Neuroimage, 52(3), 1059-1069.

23. Dennis, E. L., Jahanshad, N., McMahon, K. L., de Zubicaray, G. I., Martin, N. G., Hickie, I. B., et al. (2013). Development of brain structural connectivity between ages 12 and 30: a 4-Tesla diffusion imaging study in 439 adolescents and adults. Neuroimage, 64, 671-684.

24. Chen, Z., Liu, M., Gross, D. W., \& Beaulieu, C. (2013). Graph theoretical analysis of developmental patterns of the white matter network. Frontiers in Human Neuroscience, 7, 716.

25. Ginestet, C. E., Nichols, T. E., Bullmore, E. T., \& Simmons, A. (2011). Brain network analysis: separating cost from topology using cost-integration. PLOS ONE, 6(7), e21570.

26. Meng, X., Li, G., Jia, Y., Liu, Y., Shang, B., Liu, P., et al. (2020). Effects of dance intervention on global cognition, executive function and memory of older adults: a meta-analysis and systematic review. Aging Clinical and Experimental Research, 32(1), 7-19.

27. Foster, P. P. (2013). How does dancing promote brain reconditioning in the elderly? Frontiers in Aging Neuroscience, 5, 4.

28. Kimura, K., \& Hozumi, N. (2012). Investigating the acute effect of an aerobic dance exercise program on neuro-cognitive function in the elderly. Psychology of Sport and Exercise, 13(5), 623-629.

29. Anderson-Hanley, C., Arciero, P. J., Brickman, A. M., Nimon, J. P., Okuma, N., Westen, S. C., et al. (2012). Exergaming and older adult cognition: A cluster randomized clinical trial. American Journal of Preventive Medicine, 42(2), 109-119.

30. Voss, M. W., Weng, T. B., Burzynska, A. Z., Wong, C. N., Cooke, G. E., Clark, R., et al. (2016). Fitness, but not physical activity, is related to functional integrity of brain networks associated with aging. Neuroimage, 131, 113-125.
31. Grady, C. (2012). The cognitive neuroscience of ageing. Nature Reviews Neuroscience, 13(7), 491-505.

32. du Boisgueheneuc, F., Levy, R., Volle, E., Seassau, M., Duffau, H., Kinkingnehun, S., et al. (2006). Functions of the left superior frontal gyrus in humans: A lesion study. Brain, 129(12), $3315-3328$.

33. Menon, V. (2015). Salience network. Brain Mapping: An Encyclopedic Reference, Elsevier.

34. Weissman, D. H., Gopalakrishnan, A., Hazlett, C. J., \& Woldorff, M. G. (2005). Dorsal anterior cingulate cortex resolves conflict from distracting stimuli by boosting attention toward relevant events. Cereberal Cortex, 15(2), 229-237.

35. Colcombe, S. J., Erickson, K. I., Scalf, P. E., Kim, J. S., Prakash, R., McAuley, E., et al. (2006). Aerobic exercise training increases brain volume in aging humans. The Journals of Gerontology Series A, 61(11), 1166-1170.

36. Voss, M. W., Prakash, R. S., Erickson, K. I., Basak, C., Chaddock, L., Kim, J. S., et al. (2010). Plasticity of brain networks in a randomized intervention trial of exercise training in older adults. Frontiers in Aging Neuroscience, 2.

37. Zhu, W., Wen, W., He, Y., Xia, A., Anstey, K. J., \& Sachdev, P. (2012). Changing topological patterns in normal aging using large-scale structural networks. Neurobiology of Aging, 33(5), 899-913.

38. Wu, K., Taki, Y., Sato, K., Qi, H., Kawashima, R., \& Fukuda, H. (2013). A longitudinal study of structural brain network changes with normal aging. Frontiers in Human Neuroscience, 7, 113.

39. Pieramico, V., Esposito, R., Sensi, F., Cilli, F., Mantini, D., Mattei, P. A., et al. (2012). Combination training in aging individuals modifies functional connectivity and cognition, and is potentially affected by dopamine-related genes. PLOS ONE, 7(8), e43901.

40. Fagerholm, E. D., Hellyer, P. J., Scott, G., Leech, R., \& Sharp, D. J. (2015). Disconnection of network hubs and cognitive impairment after traumatic brain injury. Brain, 138(6), 1696-1709.

41. Taki, Y., Thyreau, B., Kinomura, S., Sato, K., Goto, R., Kawashima, R., et al. (2011). Correlations among brain gray matter volumes, age, gender, and hemisphere in healthy individuals. PLOS ONE, 6(7), e22734. 